\title{
Non-indocyanine green angiograph diagnostic criteria for polypoidal choroidal vasculopathy
}

Among Asian patients with neovascular age-related macular degeneration, the proportion of polypoidal choroidal vasculopathy (PCV) based on indocyanine green angiography (ICGA) findings has been estimated to be up to $60 \%$ in clinic-based case series. ${ }^{1,2}$ The current gold standard for diagnosing PCV requires ICGA, which is an expensive and potentially harmful procedure that may not be readily available in many ophthalmic centers.

The Asia-Pacific Ocular Imaging Society PCV Workgroup has recently published the diagnostic criteria for naïve $\mathrm{PCV}$ that do not require the use of ICGA. ${ }^{3}$ The presence of three major criteria - sub-retinal pigment epithelium ring-like structure on cross-sectional optical coherence tomography (OCT), complex retinal pigment epithelium elevation on en face OCT, and sharp-peaked pigment epithelial detachment on cross-sectional OCT - achieved an area under the receiver operating characteristic curve (AUC) of 0.90 , sensitivity of 0.75 , specificity of 0.91 , positive predictive value of 0.93 , and negative predictive value of 0.68 . When the criteria were applied by both residents and specialists, an accuracy of $82 \%$ was achieved. These diagnostic criteria provide a practical, quick, and non-invasive method for differentiating PCV from typical wet age-related macular

\section{References}

1. Kwok AK, Lai TY, Chan CW, Neoh EL, Lam DS. Polypoidal choroidal vasculopathy in Chinese patients. Br J Ophthalmol 2002;86:892-7. Crossref

2. Cheung CMG, Lai TYY, Ruamviboonsuk P, et al. Polypoidal choroidal vasculopathy: definition, pathogenesis, diagnosis, degeneration, especially in clinical settings that ICGA is not accessible. It is supported that ICGA can be dispensed with under most conditions. When en face OCT was not available, the combination of the remaining two spectraldomain OCT-based criteria achieved an AUC of 0.82. The absence of these diagnostic criteria did not exclude the diagnosis of PCV.

Based on multimodal imaging and histologic studies, the terms 'polypoidal lesion' and 'branching neovascular network' were recommended, instead of 'polyp' and 'branching vascular network', respectively, which are based on ICGA appearance.

Alvin KH Kwok, MD (HK), MD (CUHK), PhD (HK), FRCS (UK), FRCOphth (UK), FHKAM (Ophth), PostGrad DipEpidem \& Biostat (CUHK), MBBS (HK)

Department of Ophthalmology, The Hong Kong Sanatorium and Hospital, Hong Kong

Correspondence and reprint requests:

Dr Alvin KH Kwok, Department of Ophthalmology, 4/F, Li Shu Fan Block, The Hong Kong Sanatorium and Hospital, 2 Village Road, Hong Kong.

Email:alvinkhkwok@netvigator.com 